\title{
Synthesis and Antimicrobial Activity of a New Class of Sulfone Linked Bisheterocycles
}

\author{
Venkatapuram Padmavathi', Thunga Radha Lakshmi, \\ Bhumireddy Chinnachennaiahgari Venkatesh, Konda Mahesh \\ Department of Chemistry, Sri Venkateswara University, Tirupati, India \\ E-mail: vkpuram2001@yahoo.com \\ Received June 1, 2011; revised July 20, 2011; accepted August 1, 2011
}

\begin{abstract}
A new and novel class of bis(heterocycles) viz., bis pyrroles, pyrrolyl pyrazoles and pyrrolyl isoxazoles are prepared from 1-aroyl-2-styrylsulfonylethenes by 1,3-dipolar cycloaddition of tosylmethyl isocyanide, diazomethane, nitrile imines and nitrile oxides. The lead compounds are screened for antimicrobial activity.
\end{abstract}

Keywords: 1-Aroyl-2-Styrylsulfonylethene, 1,3-Dipolar Cycloaddition, Chloramine-T, Antimicrobial Activity

\section{Introduction}

In the last few decades the chemistry of five-membered heterocycles particularly pyrroles, pyrazolines and isoxazolines has received considerable attention owing to their synthetic and effective biological importance. Increasing evidence suggests that pyrazoline derivatives possess a broad spectrum of biological activities such as antidepressant, anticonvulsant, psychoanalytic, antihypotensive and monamine oxidase inhibitory activities [1, 2]. In fact, Celecoxib, a pyrazole derivative and Valdecoxib an isoxazole derivative are extensively used as anti-inflammatory drugs [3]. Besides, pyrrole carboxylates exhibit antibiotic, antiviral and oncolytic properties [4-8]. Hence, it is thought that a worthwhile programme would be to prepare molecules having both pyrrole and pyrazole/isoxazole units. Literature evidenced the synthesis of 3,4-disubstituted pyrroles by cyclocondensation of Michael acceptors with tosylmethyl isocyanide (TosMIC) [9]. Pyrroles have also been prepared by PaalKnorr condensation of alkyl and aryl amines with 1,4diketones [10-13]. Similarly, among different methods for the synthesis of pyrazolines and isoxazolines, 1,3dipolar cycloaddition of an ylide onto an alkene in a $3+$ 2 manner is a facile one [14,15]. Indeed, diazomethane, nitrile imines and nitrile oxides have been used extensively as reactive intermediates. The nitrile imines and nitrile oxides can be generated by dehydrogenation of araldehyde phenylhydrazones and araldoximes with lead tetraacetate [16], mercuric acetate [17], 1-chlorobenzotriazole [18], chloramine-T [19-22] etc. The present communication deals with the synthesis of sulfone linked bis heterocycles having a pyrrole in combination with a pyrazole or an isoxazole unit.

\section{Results and Discussion}

The synthetic scheme is based on the reactivity of 1-aroyl-2-styrylsulfonylethene (1) towards 1,3-dipolar reagents viz., TosMIC, diazomethane, nitrile imines, nitrile oxides. When compound (1) is treated with TosMIC in the presence of sodium hydride in a mixture of ether and DMSO, the reaction took place regioselectively resulting in a mixture of compounds in 3:1 ratio. They are identified as 4-aroyl-3-(phenylethenesulfonyl)- $1 H$-pyrrole (2) and 4-aroyl-3-(4'-phenyl-1' $H$-pyrrol-3'-ylsulfonyl)-1H-pyrrole (3) in major and minor amounts, respectively (Scheme 1, Table 1). However, repetition of this reaction with excess TosMIC resulted in $\mathbf{3}$ only. The latter compound is also obtained by treating 2 with one equivalent of TosMIC. The ${ }^{1} \mathrm{H}$ NMR spectrum of $\mathbf{2 a}$ showed two singlets at $\delta 7.01$ and $8.02 \mathrm{ppm}$ for $\mathrm{C}_{2}-\mathrm{H}$, and $\mathrm{C}_{5}-\mathrm{H}$ of pyrrole ring protons. Two doublets are observed at 6.79 and $7.48 \mathrm{ppm}$ corresponding to olefin protons in addition to the signals of the aromatic protons. The coupling constants value $(J=17.8 \mathrm{~Hz})$ indicates that they are in trans geometry. Compound 3a exhibited three singlets at $\delta 6.89\left(\mathrm{C}_{2}-\mathrm{H} \& \mathrm{C}_{2},-\mathrm{H}\right), 6.96\left(\mathrm{C}_{5},-\mathrm{H}\right)$ and 8.04 $\left(\mathrm{C}_{5}-\mathrm{H}\right) \mathrm{ppm}$ apart from signals due to aromatic protons (Table 2).

The olefin moiety in $\mathbf{2}$ is used to develop different het- 


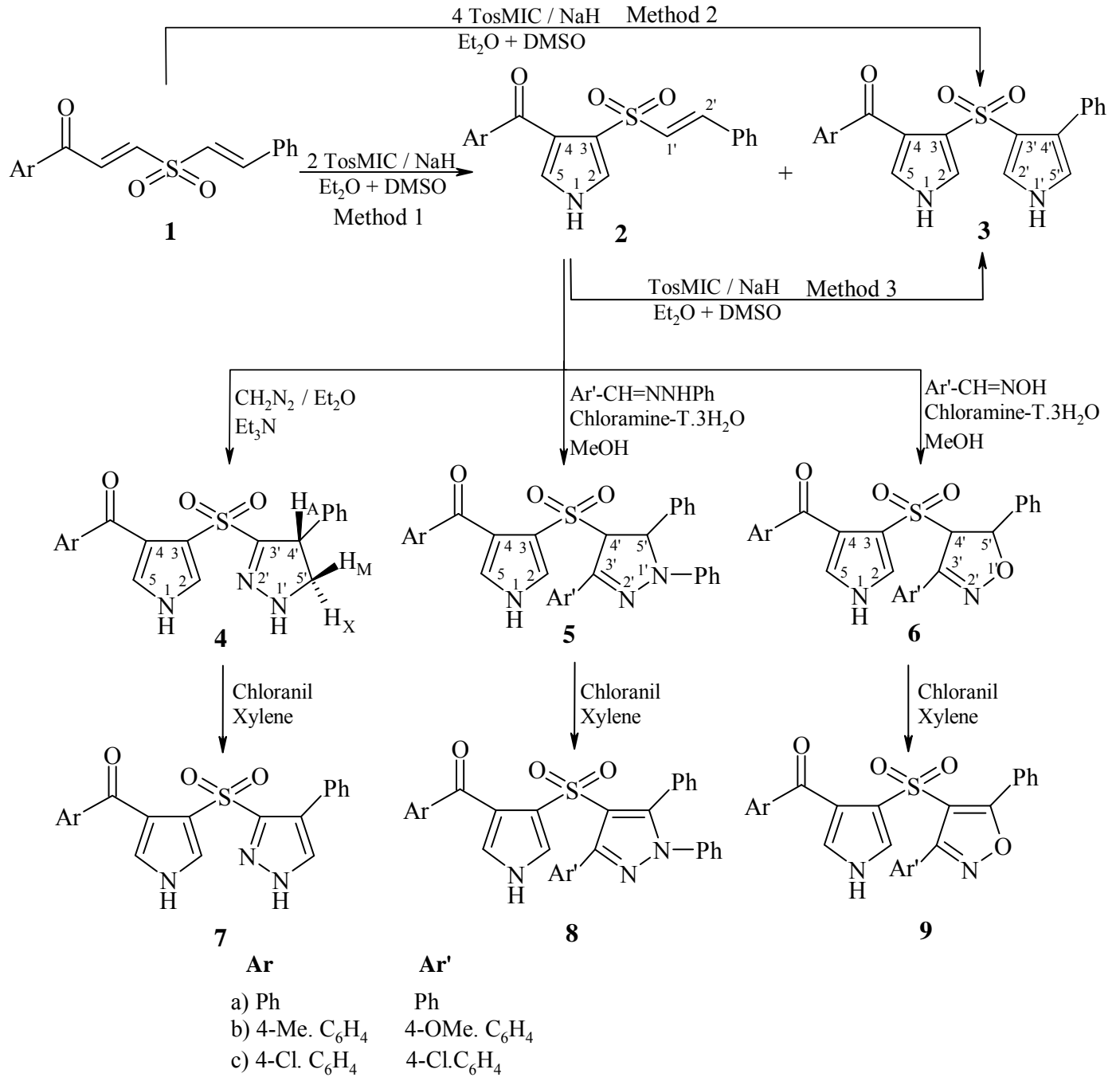

Scheme 1. Synthesis of bis heterocycles.

erocyclic rings such as pyrazoles and isoxazoles. Treatment of 2 with diazomethane at $-20^{\circ} \mathrm{C}$ to $-15^{\circ} \mathrm{C}$ for $48 \mathrm{~h}$ gave a solid which is identified as 4-aroyl-3-(4'-phenyl-4',5'-dihydro-1' $H$-pyrazol-3'-ylsulfonyl)-1 $H$-pyrrole (4) by spectral analysis. The ${ }^{1} \mathrm{H}$ NMR spectrum of $4 \mathrm{a}$ showed an AMX splitting pattern for the pyrazoline ring protons exhibiting three double doublets at $\delta 4.54\left(\mathrm{H}_{\mathrm{A}}\right)$, $3.99\left(\mathrm{H}_{\mathrm{M}}\right)$ and $3.64\left(\mathrm{H}_{\mathrm{X}}\right)$ ppm, apart from the signals of aromatic and pyrrole ring protons. The observed coupling constant values $J_{\mathrm{AM}}=11.6, J_{\mathrm{AX}}=5.1$ and $J_{\mathrm{MX}}=$ $10.3 \mathrm{~Hz}$ indicates that $\mathrm{H}_{\mathrm{A}}$ and $\mathrm{H}_{\mathrm{M}}$ are cis, $\mathrm{H}_{\mathrm{A}}$ and $\mathrm{H}_{\mathrm{X}}$ are trans and $\mathrm{H}_{M}$ and $\mathrm{H}_{\mathrm{X}}$ are geminal (Table 2). Similarly, 1,3-dipolar cycloaddition reaction of nitrile imines and nitrile oxides generated from araldehyde phenylhydrazones and araldoximes to 2 resulted in 4-aroyl-3-(1',5'diphenyl-3'-aryl-4',5'-dihydro-1' $H$-pyrazol-4'-ylsulfonyl)$1 H$-pyrrole (5) and 4-aroyl-3-(3'-aryl-5'-phenyl-4',5'- dihydroisoxazol-4'-ylsulfonyl)-1 $H$-pyrrole (6), respectively (Scheme 1, Table 1). The ${ }^{1} \mathrm{H}-\mathrm{NMR}$ spectra of $\mathbf{5 a}$ and 6a displayed two doublets at $\delta 5.19,5.22$ and 5.58, $5.66 \mathrm{ppm}$, which are assigned to $\mathrm{C}_{4},-\mathrm{H}$ and $\mathrm{C}_{5}, \mathrm{H}$, the two methine protons of the pyrazoline and isoxazo- line rings. The $J$ values $(J=6.3 \& 6.4 \mathrm{~Hz})$ shows that they are in trans geometry.

The olefin moiety in $\mathbf{2}$ is used to develop different heterocyclic rings such as pyrazoles and isoxazoles. Treatment of 2 with diazomethane at $-20^{\circ} \mathrm{C}$ to $-15^{\circ} \mathrm{C}$ for $48 \mathrm{~h}$ gave a solid which is identified as 4-aroyl-3(4'-phenyl-4',5' -dihydro-1' $H$-pyrazol-3'-ylsulfonyl)- $1 H$ pyrrole (4) by spectral analysis. The ${ }^{1} \mathrm{H}$ NMR spectrum of 4a showed an AMX splitting pattern for the pyrazoline ring protons exhibiting three double doublets at $\delta$ $4.54\left(\mathrm{H}_{\mathrm{A}}\right), 3.99\left(\mathrm{H}_{\mathrm{M}}\right)$ and $3.64\left(\mathrm{H}_{\mathrm{X}}\right) \mathrm{ppm}$, apart from the signals of aromatic and pyrrole ring protons. The observed coupling constant values $J_{\mathrm{AM}}=11.6, J_{\mathrm{AX}}=5.1$ 
Table 1. Physical characterization data of compounds 2-9.

\begin{tabular}{|c|c|c|c|c|c|c|}
\hline \multirow{2}{*}{ Compd } & \multirow{2}{*}{ m.p. $\left({ }^{\circ} \mathrm{C}\right)$} & \multirow{2}{*}{ Yield (\%) } & \multirow{2}{*}{ Mol. Formula (Mol. wt.) } & \multicolumn{3}{|c|}{ Found \% (Calcd) } \\
\hline & & & & $\mathrm{C}$ & $\mathrm{H}$ & $\mathrm{N}$ \\
\hline $2 a$ & $178-80$ & 56 & $\mathrm{C}_{19} \mathrm{H}_{15} \mathrm{NO}_{3} \mathrm{~S}$ (337.39) & $67.71(67.64)$ & $5.01(4.48)$ & $4.22(4.15)$ \\
\hline $2 \mathbf{b}$ & $169-71$ & 54 & $\mathrm{C}_{20} \mathrm{H}_{17} \mathrm{NO}_{3} \mathrm{~S}(351.42)$ & $68.50(68.36)$ & $4.82(4.88)$ & $4.04(3.99)$ \\
\hline 2c & $211-13$ & 60 & $\mathrm{C}_{19} \mathrm{H}_{14} \mathrm{ClNO}_{3} \mathrm{~S}$ (371.84) & $61.32(61.37)$ & $3.83(3.79)$ & $3.81(3.77)$ \\
\hline 3a & $185-87$ & $16,70^{\mathrm{a}}, 72^{\mathrm{b}}$ & $\mathrm{C}_{21} \mathrm{H}_{16} \mathrm{~N}_{2} \mathrm{O}_{3} \mathrm{~S}$ (376.43) & $67.12(67.00)$ & $4.27(4.28)$ & $7.52(7.44)$ \\
\hline $3 \mathbf{b}$ & $192-94$ & $10,64^{\mathrm{a}}, 71^{\mathrm{b}}$ & $\mathrm{C}_{22} \mathrm{H}_{18} \mathrm{~N}_{2} \mathrm{O}_{3} \mathrm{~S}$ (390.45) & $67.75(67.67)$ & $4.69(4.65)$ & $7.14(7.17)$ \\
\hline 3c & $220-22$ & $12,68^{\mathrm{a}}, 76^{\mathrm{b}}$ & $\mathrm{C}_{21} \mathrm{H}_{15} \mathrm{ClN}_{2} \mathrm{O}_{3} \mathrm{~S}$ (410.87) & $61.46(61.39)$ & $3.70(3.68)$ & $6.85(6.82)$ \\
\hline $4 a$ & $196-98$ & 67 & $\mathrm{C}_{20} \mathrm{H}_{17} \mathrm{~N}_{3} \mathrm{O}_{3} \mathrm{~S}$ (379.43) & $63.26(63.31)$ & $4.57(4.52)$ & $11.11(11.07)$ \\
\hline $4 b$ & $207-09$ & 70 & $\mathrm{C}_{21} \mathrm{H}_{19} \mathrm{~N}_{3} \mathrm{O}_{3} \mathrm{~S}$ (393.46) & $64.18(64.10)$ & $4.84(4.87)$ & $10.72(10.68)$ \\
\hline $4 c$ & $235-37$ & 73 & $\mathrm{C}_{20} \mathrm{H}_{16} \mathrm{ClN}_{3} \mathrm{O}_{3} \mathrm{~S}(413.88)$ & $58.00(58.04)$ & $3.94(3.90)$ & $10.22(10.15)$ \\
\hline $5 a$ & $225-27$ & 72 & $\mathrm{C}_{32} \mathrm{H}_{25} \mathrm{~N}_{3} \mathrm{O}_{3} \mathrm{~S}$ (531.62) & $72.37(72.30)$ & $4.80(4.74)$ & $7.96(7.90)$ \\
\hline $5 \mathbf{b}$ & $212-14$ & 69 & $\mathrm{C}_{34} \mathrm{H}_{29} \mathrm{~N}_{3} \mathrm{O}_{4} \mathrm{~S}(575.68)$ & $70.88(70.94)$ & $5.05(5.08)$ & $7.36(7.30)$ \\
\hline $5 c$ & $243-45$ & 75 & $\mathrm{C}_{32} \mathrm{H}_{23} \mathrm{Cl}_{2} \mathrm{~N}_{3} \mathrm{O}_{3} \mathrm{~S}(600.51)$ & $64.06(64.00)$ & $3.82(3.86)$ & $7.05(7.00)$ \\
\hline 6a & $202-04$ & 76 & $\mathrm{C}_{26} \mathrm{H}_{20} \mathrm{~N}_{2} \mathrm{O}_{4} \mathrm{~S}(456.51)$ & $68.50(68.41)$ & $4.44(4.42)$ & $6.10(6.14)$ \\
\hline $6 \mathbf{b}$ & $215-17$ & 74 & $\mathrm{C}_{28} \mathrm{H}_{24} \mathrm{~N}_{2} \mathrm{O}_{5} \mathrm{~S}(500.57)$ & $67.14(67.18)$ & $4.82(4.83)$ & $5.63(5.60)$ \\
\hline 6c & $231-33$ & 78 & $\mathrm{C}_{26} \mathrm{H}_{18} \mathrm{Cl}_{2} \mathrm{~N}_{2} \mathrm{O}_{4} \mathrm{~S}(525.40)$ & $59.51(59.44)$ & $3.50(3.45)$ & $5.38(5.33)$ \\
\hline $7 a$ & $204-06$ & 65 & $\mathrm{C}_{20} \mathrm{H}_{15} \mathrm{~N}_{3} \mathrm{O}_{3} \mathrm{~S}$ (377.42) & $63.73(63.65)$ & $4.04(4.01)$ & $11.26(11.13)$ \\
\hline $7 \mathbf{b}$ & $214-16$ & 62 & $\mathrm{C}_{21} \mathrm{H}_{17} \mathrm{~N}_{3} \mathrm{O}_{3} \mathrm{~S}$ (391.44) & $64.49(64.43)$ & $4.40(4.38)$ & $10.80(10.73)$ \\
\hline $7 c$ & $247-49$ & 67 & $\mathrm{C}_{20} \mathrm{H}_{14} \mathrm{ClN}_{3} \mathrm{O}_{3} \mathrm{~S}$ (411.86) & $58.38(58.32)$ & $3.48(3.43)$ & $10.30(10.20)$ \\
\hline $8 a$ & $237-39$ & 64 & $\mathrm{C}_{32} \mathrm{H}_{23} \mathrm{~N}_{3} \mathrm{O}_{3} \mathrm{~S}(529.61)$ & $72.70(72.57)$ & $4.37(4.38)$ & $8.00(7.93)$ \\
\hline $8 \mathbf{b}$ & $242-44$ & 70 & $\mathrm{C}_{34} \mathrm{H}_{27} \mathrm{~N}_{3} \mathrm{O}_{4} \mathrm{~S}(573.66)$ & $71.29(71.19)$ & $4.79(4.74)$ & $7.28(7.32)$ \\
\hline $8 c$ & $269-71$ & 68 & $\mathrm{C}_{32} \mathrm{H}_{21} \mathrm{Cl}_{2} \mathrm{~N}_{3} \mathrm{O}_{3} \mathrm{~S}$ (598.50) & $64.31(64.22)$ & $3.51(3.54)$ & $7.08(7.02)$ \\
\hline 9a & $211-13$ & 66 & $\mathrm{C}_{26} \mathrm{H}_{18} \mathrm{~N}_{2} \mathrm{O}_{4} \mathrm{~S}$ (454.50) & $68.78(68.71)$ & $4.03(3.99)$ & $6.13(6.16)$ \\
\hline $9 b$ & $227-29$ & 63 & $\mathrm{C}_{28} \mathrm{H}_{22} \mathrm{~N}_{2} \mathrm{O}_{5} \mathrm{~S}(498.55)$ & $67.48(67.46)$ & $4.48(4.45)$ & $5.57(5.62)$ \\
\hline 9c & $246-48$ & 72 & $\mathrm{C}_{26} \mathrm{H}_{16} \mathrm{Cl}_{2} \mathrm{~N}_{2} \mathrm{O}_{4} \mathrm{~S}$ (523.39) & $59.75(59.66)$ & $3.08(3.06)$ & $5.41(5.35)$ \\
\hline
\end{tabular}

${ }^{\mathrm{a}}$ Yield in Method-2; ${ }^{\mathrm{b}}$ Yield in Method-3 
Table 2. IR, ${ }^{1} \mathrm{H}$ and ${ }^{13} \mathrm{C}$ NMR spectral characterization data of compounds 2-9.

\begin{tabular}{|c|c|c|c|}
\hline Compd & IR $(\mathrm{KBr}) \mathrm{cm}^{-1}$ & ${ }^{1} \mathrm{H} \operatorname{NMR}(\delta, \mathrm{ppm})(J$ in $\mathrm{Hz})$ & ${ }^{13} \mathrm{C}$ NMR ( $\delta$, ppm) \\
\hline $2 a$ & $\begin{array}{c}3326(\mathrm{NH}), 1660 \\
(\mathrm{C}=\mathrm{O}), 1641 \\
(\mathrm{C}=\mathrm{C}), 1334,1131 \\
\left(\mathrm{SO}_{2}\right)\end{array}$ & $\begin{array}{c}6.79\left(\mathrm{~d}, 1 \mathrm{H}, \mathrm{C}_{1},-\mathrm{H}, J=17.8 \mathrm{~Hz}\right), 7.01\left(\mathrm{~s}, 1 \mathrm{H}, \mathrm{C}_{2}-\mathrm{H}\right) \\
7.48\left(\mathrm{~d}, 1 \mathrm{H}, \mathrm{C}_{2},-\mathrm{H}, J=17.8 \mathrm{~Hz}\right), 7.14-7.85(\mathrm{~m}, 10 \mathrm{H} \\
\text { Ar-H), } 8.02\left(\mathrm{~s}, 1 \mathrm{H}, \mathrm{C}_{5}-\mathrm{H}\right), 10.79(\mathrm{bs}, 1 \mathrm{H}, \mathrm{NH})\end{array}$ & $\begin{array}{c}108.6(\mathrm{C}-3), 117.3(\mathrm{C}-4), 120.0\left(\mathrm{C}-1^{\prime}\right), 121.4(\mathrm{C}-2), \\
136.9(\mathrm{C}-5), 137.7\left(\mathrm{C}-2^{\prime}\right), 189.4(\mathrm{C}=\mathrm{O}), 128.4,129.6, \\
130.9,131.5,132.9,133.7,134.4 \text { (aromatic carbons) }\end{array}$ \\
\hline $2 c$ & $\begin{array}{c}3335(\mathrm{NH}), 1664 \\
(\mathrm{C}=\mathrm{O}), 1635 \\
(\mathrm{C}=\mathrm{C}), 1337,1130 \\
\left(\mathrm{SO}_{2}\right)\end{array}$ & $\begin{array}{c}6.77\left(\mathrm{~d}, 1 \mathrm{H}, \mathrm{C}_{1},-\mathrm{H}, J=17.7 \mathrm{~Hz}\right), 7.07\left(\mathrm{~s}, 1 \mathrm{H}, \mathrm{C}_{2}-\mathrm{H}\right) \\
7.46\left(\mathrm{~d}, 1 \mathrm{H}, \mathrm{C}_{2}-\mathrm{H}, J=17.7 \mathrm{~Hz}\right), 7.21-7.89(\mathrm{~m}, 9 \mathrm{H} \\
\mathrm{Ar}-\mathrm{H}), 8.01\left(\mathrm{~s}, 1 \mathrm{H}, \mathrm{C}_{5}-\mathrm{H}\right), 10.81(\mathrm{bs}, 1 \mathrm{H}, \mathrm{NH})\end{array}$ & $\begin{array}{c}108.8(\mathrm{C}-3), 118.3(\mathrm{C}-4), 121.2\left(\mathrm{C}-1^{\prime}\right), 121.3(\mathrm{C}-2), \\
136.2(\mathrm{C}-5), 137.4\left(\mathrm{C}-2^{\prime}\right), 189.6(\mathrm{C}=\mathrm{O}), 128.2,129.6, \\
130.3,131.7,133.8,134.9,135.6 \text { (aromatic carbons) }\end{array}$ \\
\hline 3a & $\begin{array}{c}3329(\mathrm{NH}), 1662 \\
(\mathrm{C}=\mathrm{O}), 1331,1129 \\
\left(\mathrm{SO}_{2}\right)\end{array}$ & $\begin{array}{c}6.89\left(\mathrm{~s}, 2 \mathrm{H}, \mathrm{C}_{2}-\mathrm{H} \text { and } \mathrm{C}_{2},-\mathrm{H}\right), 6.96\left(\mathrm{~s}, 1 \mathrm{H}, \mathrm{C}_{5},-\mathrm{H}\right), 7.25 \\
-7.78(\mathrm{~m}, 10 \mathrm{H}, \mathrm{Ar}-\mathrm{H}), 8.04\left(\mathrm{~s}, 1 \mathrm{H}, \mathrm{C}_{5}-\mathrm{H}\right), 10.42(\mathrm{bs} \\
2 \mathrm{H}, \mathrm{NH})\end{array}$ & $\begin{array}{c}105.3\left(\mathrm{C}-4{ }^{\prime}\right), 109.6\left(\mathrm{C}-3 \text { and } \mathrm{C}-3^{\prime}\right), 115.3(\mathrm{C}-4) \\
118.5\left(\mathrm{C}-2 \text { and } \mathrm{C}-2^{\prime}\right), 119.7\left(\mathrm{C}-5^{\prime}\right), 138.2(\mathrm{C}-5), \\
188.4(\mathrm{C}=\mathrm{O}), 128.2,129.5,130.6,131.3,132.9 \\
133.7,134.6,135.2 \text { (aromatic carbons) }\end{array}$ \\
\hline $3 \mathbf{b}$ & $\begin{array}{c}3324(\mathrm{NH}), 1668 \\
(\mathrm{C}=\mathrm{O}), 1335,1139 \\
\left(\mathrm{SO}_{2}\right)\end{array}$ & $\begin{array}{c}2.25\left(\mathrm{~s}, 3 \mathrm{H}, \mathrm{Ar}-\mathrm{CH}_{3}\right), 6.85\left(\mathrm{~s}, 2 \mathrm{H}, \mathrm{C}_{2}-\mathrm{H} \text { and } \mathrm{C}_{2},-\mathrm{H}\right) \\
6.99\left(\mathrm{~s}, 1 \mathrm{H}, \mathrm{C}_{5}, \mathrm{H}\right), 7.19-7.74(\mathrm{~m}, 9 \mathrm{H}, \mathrm{Ar}-\mathrm{H}), 8.02(\mathrm{~s} \\
\left.1 \mathrm{H}, \mathrm{C}_{5}-\mathrm{H}\right), 10.32(\mathrm{bs}, 2 \mathrm{H}, \mathrm{NH})\end{array}$ & 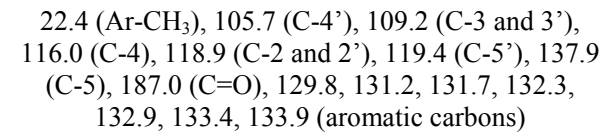 \\
\hline $3 c$ & $\begin{array}{c}3330(\mathrm{NH}), 1666 \\
(\mathrm{C}=\mathrm{O}), 1329,1126 \\
\left(\mathrm{SO}_{2}\right)\end{array}$ & $\begin{array}{c}7.13\left(\mathrm{~s}, 2 \mathrm{H}, \mathrm{C}_{2}-\mathrm{H} \text { and } \mathrm{C}_{2},-\mathrm{H}\right), 7.35\left(\mathrm{~s}, 1 \mathrm{H}, \mathrm{C}_{5},-\mathrm{H}\right), 7.26 \\
-7.99(\mathrm{~m}, 9 \mathrm{H}, \mathrm{Ar}-\mathrm{H}), 8.12\left(\mathrm{~s}, 1 \mathrm{H}, \mathrm{C}_{5}-\mathrm{H}\right), 10.55(\mathrm{bs} \\
2 \mathrm{H}, \mathrm{NH})\end{array}$ & $\begin{array}{c}104.8\left(\mathrm{C}-4{ }^{\prime}\right), 108.3\left(\mathrm{C}-3 \text { and } \mathrm{C}-3{ }^{\prime}\right), 115.3(\mathrm{C}-4), \\
118.5\left(\mathrm{C}-2 \text { and } \mathrm{C}-2^{\prime}\right), 121.8\left(\mathrm{C}-5^{\prime}\right), 138.3(\mathrm{C}-5), \\
187.5(\mathrm{C}=\mathrm{O}), 128.3,129.1,129.8,130.6,132.4 \\
133.0,137.4,138.3 \text { (aromatic carbons) }\end{array}$ \\
\hline $4 \mathbf{b}$ & $\begin{array}{c}3342(\mathrm{NH}), 1662 \\
(\mathrm{C}=\mathrm{O}), 1576 \\
(\mathrm{C}=\mathrm{N}), 1317,1140 \\
\left(\mathrm{SO}_{2}\right)\end{array}$ & $\begin{array}{c}2.28\left(\mathrm{~s}, 3 \mathrm{H}, \mathrm{Ar}-\mathrm{CH}_{3}\right), 3.85\left(\mathrm{dd}, 1 \mathrm{H}, \mathrm{H}_{\mathrm{X}}\right), 4.22(\mathrm{dd}, 1 \mathrm{H} \\
\left.\mathrm{H}_{\mathrm{M}}, J_{\mathrm{MX}}=8.2 \mathrm{~Hz}\right), 4.54\left(\mathrm{dd}, 1 \mathrm{H}, \mathrm{H}_{\mathrm{A}}, J_{\mathrm{AM}}=12.0 \mathrm{~Hz}\right. \\
\left.J_{\mathrm{AX}}=4.3 \mathrm{~Hz}\right), 6.94\left(\mathrm{~s}, 1 \mathrm{H}, \mathrm{C}_{2}-\mathrm{H}\right), 7.22-7.64(\mathrm{~m}, 9 \mathrm{H} \\
\mathrm{Ar}-\mathrm{H}), 7.95\left(\mathrm{~s}, 1 \mathrm{H}, \mathrm{C}_{5}-\mathrm{H}\right), 8.89(\mathrm{bs}, 1 \mathrm{H}, \mathrm{NH}), 10.36 \\
(\mathrm{bs}, 1 \mathrm{H}, \mathrm{NH})\end{array}$ & 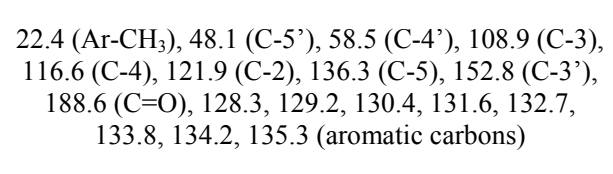 \\
\hline $4 c$ & $\begin{array}{c}3320(\mathrm{NH}), 1669 \\
(\mathrm{C}=\mathrm{O}), 1569 \\
(\mathrm{C}=\mathrm{N}), 1321,1131 \\
\left(\mathrm{SO}_{2}\right)\end{array}$ & $\begin{array}{c}3.83\left(\mathrm{dd}, 1 \mathrm{H}, \mathrm{H}_{\mathrm{X}}\right), 4.27\left(\mathrm{dd}, 1 \mathrm{H}, \mathrm{H}_{\mathrm{M}}, J_{\mathrm{MX}}=8.0 \mathrm{~Hz}\right) \\
4.56\left(\mathrm{dd}, 1 \mathrm{H}, \mathrm{H}_{\mathrm{A}}, J_{\mathrm{AM}}=12.1 \mathrm{~Hz}, J_{\mathrm{AX}}=4.5 \mathrm{~Hz}\right), 6.97 \\
\left(\mathrm{~s}, 1 \mathrm{H}, \mathrm{C}_{2}-\mathrm{H}\right), 7.29-7.74(\mathrm{~m}, 9 \mathrm{H}, \mathrm{Ar}-\mathrm{H}), 7.93(\mathrm{~s}, 1 \mathrm{H} \\
\left.\mathrm{C}_{5}-\mathrm{H}\right), 8.94(\mathrm{bs}, 1 \mathrm{H}, \mathrm{NH}), 10.39(\mathrm{bs}, 1 \mathrm{H}, \mathrm{NH})\end{array}$ & $\begin{array}{c}48.7\left(\mathrm{C}^{\prime} 5^{\prime}\right), 59.2\left(\mathrm{C}^{\prime} 4^{\prime}\right), 108.1(\mathrm{C}-3), 116.9(\mathrm{C}-4), \\
122.6(\mathrm{C}-2), 136.9(\mathrm{C}-5), 153.2\left(\mathrm{C}-3^{\prime}\right), 187.1(\mathrm{C}=\mathrm{O}), \\
128.6,129.4,130.8,131.7,132.6,133.2,135.6,138.1 \\
\text { (aromatic carbons) }\end{array}$ \\
\hline $5 a$ & $\begin{array}{c}3332(\mathrm{NH}), 1682 \\
(\mathrm{C}=\mathrm{O}), 1572 \\
(\mathrm{C}=\mathrm{N}), 1328,1123 \\
\left(\mathrm{SO}_{2}\right)\end{array}$ & $\begin{array}{c}5.19\left(\mathrm{~d}, 1 \mathrm{H}, \mathrm{C}_{4},-\mathrm{H}, J=6.3 \mathrm{~Hz}\right), 5.58\left(\mathrm{~d}, 1 \mathrm{H}, \mathrm{C}_{5},-\mathrm{H}, J=\right. \\
6.3 \mathrm{~Hz}), 6.99\left(\mathrm{~s}, 1 \mathrm{H}, \mathrm{C}_{2}-\mathrm{H}\right), 7.21-7.74(\mathrm{~m}, 20 \mathrm{H}, \mathrm{Ar} \& \\
\left.\mathrm{Ar}^{\prime}-\mathrm{H}\right), 8.01\left(\mathrm{~s}, 1 \mathrm{H}, \mathrm{C}_{5}-\mathrm{H}\right), 10.71(\mathrm{bs}, 1 \mathrm{H}, \mathrm{NH})\end{array}$ & $\begin{array}{c}61.9\left(\mathrm{C}-4^{\prime}\right), 82.8\left(\mathrm{C}-5^{\prime}\right), 108.9(\mathrm{C}-3), 117.4(\mathrm{C}-4) \\
121.9(\mathrm{C}-2), 136.2(\mathrm{C}-5), 155.2\left(\mathrm{C}-3^{\prime}\right), 188.4(\mathrm{C}=\mathrm{O}) \\
127.3,128.1,128.6,129.2,130.2,131.2,132.7 \\
133.4,134.6,136.3 \text { (aromatic carbons) }\end{array}$ \\
\hline $5 \mathbf{b}$ & $\begin{array}{c}3336(\mathrm{NH}), 1678 \\
(\mathrm{C}=\mathrm{O}), 1564 \\
(\mathrm{C}=\mathrm{N}), 1335,1133 \\
\left(\mathrm{SO}_{2}\right)\end{array}$ & $\begin{array}{c}2.25\left(\mathrm{~s}, 3 \mathrm{H}, \mathrm{Ar}-\mathrm{CH}_{3}\right), 3.72\left(\mathrm{~s}, 3 \mathrm{H}, \mathrm{OCH}_{3}\right), 5.23(\mathrm{~d}, 1 \mathrm{H}, \\
\left.\mathrm{C}_{4},-\mathrm{H}, J=6.5 \mathrm{~Hz}\right), 5.54\left(\mathrm{~d}, 1 \mathrm{H}, \mathrm{C}_{5},-\mathrm{H}, J=6.5 \mathrm{~Hz}\right) \\
6.94\left(\mathrm{~s}, 1 \mathrm{H}, \mathrm{C}_{2}-\mathrm{H}\right), 7.29-7.71(\mathrm{~m}, 18 \mathrm{H}, \mathrm{Ar} \& \mathrm{Ar}-\mathrm{H}) \\
7.98\left(\mathrm{~s}, 1 \mathrm{H}, \mathrm{C}_{5}-\mathrm{H}\right), 10.67(\mathrm{bs}, 1 \mathrm{H}, \mathrm{NH})\end{array}$ & 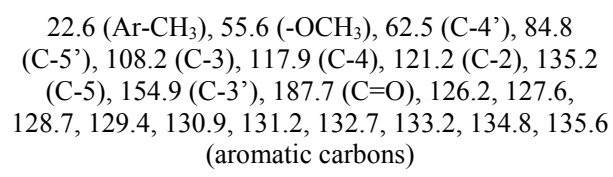 \\
\hline $5 c$ & $\begin{array}{c}3347(\mathrm{NH}), 1684 \\
(\mathrm{C}=\mathrm{O}), 1573 \\
(\mathrm{C}=\mathrm{N}), 1331,1135 \\
\left(\mathrm{SO}_{2}\right)\end{array}$ & $\begin{array}{c}5.27\left(\mathrm{~d}, 1 \mathrm{H}, \mathrm{C}_{4},-\mathrm{H}, J=6.8 \mathrm{~Hz}\right), 5.64\left(\mathrm{~d}, 1 \mathrm{H}, \mathrm{C}_{5},-\mathrm{H}, J=\right. \\
6.8 \mathrm{~Hz}), 6.98\left(\mathrm{~s}, 1 \mathrm{H}, \mathrm{C}_{2}-\mathrm{H}\right), 7.25-7.88(\mathrm{~m}, 18 \mathrm{H}, \mathrm{Ar} \& \\
\mathrm{Ar}-\mathrm{H}), 8.03\left(\mathrm{~s}, 1 \mathrm{H}, \mathrm{C}_{5}-\mathrm{H}\right), 10.64(\mathrm{bs}, 1 \mathrm{H}, \mathrm{NH})\end{array}$ & $\begin{array}{c}63.1\left(\mathrm{C}-4^{\prime}\right), 83.2\left(\mathrm{C}-5^{\prime}\right), 108.5(\mathrm{C}-3), 117.5(\mathrm{C}-4) \\
121.8(\mathrm{C}-2), 135.6(\mathrm{C}-5), 155.5\left(\mathrm{C}-3^{\prime}\right), 188.6(\mathrm{C}=\mathrm{O}) \\
127.2,128.6,129.2,129.9,130.5,131.8,132.3 \\
133.1,135.2,137.1 \text { (aromatic carbons) }\end{array}$ \\
\hline
\end{tabular}


$6 a$

$3340(\mathrm{NH}), 1662$

$(\mathrm{C}=\mathrm{O}), 1577$

$(\mathrm{C}=\mathrm{N}), 1336,1131$

$\left(\mathrm{SO}_{2}\right)$

$3337(\mathrm{NH}), 1669$

$(\mathrm{C}=\mathrm{O}), 1581$

6b

$(\mathrm{C}=\mathrm{N}), 1329,1130$ $\left(\mathrm{SO}_{2}\right)$

$3332(\mathrm{NH}), 1667$

$(\mathrm{C}=\mathrm{O}), 1570$

$6 c$

$(\mathrm{C}=\mathrm{N}), 1339,1142$, $\left(\mathrm{SO}_{2}\right)$

$3339(\mathrm{NH}), 1656$ $(\mathrm{C}=\mathrm{O}), 1632$

$(\mathrm{C}=\mathrm{C}), 1564$

$(\mathrm{C}=\mathrm{N}), 1337,1121$ $\left(\mathrm{SO}_{2}\right)$

$3328(\mathrm{NH}), 1668$ $(\mathrm{C}=\mathrm{O}), 1644$ $(\mathrm{C}=\mathrm{C}), 1574$

$(\mathrm{C}=\mathrm{N}), 1326,1138$ $\left(\mathrm{SO}_{2}\right)$

$3336(\mathrm{NH}), 1666$ $(\mathrm{C}=\mathrm{O}), 1640$ $(\mathrm{C}=\mathrm{C}), 1567$

$(\mathrm{C}=\mathrm{N}), 1330,1122$ $\left(\mathrm{SO}_{2}\right)$

$3331(\mathrm{NH}), 1658$ $(\mathrm{C}=\mathrm{O}), 1637$ $(\mathrm{C}=\mathrm{C}), 1578$

$(\mathrm{C}=\mathrm{N}), 1335,1126$ $\left(\mathrm{SO}_{2}\right)$

$3338(\mathrm{NH}), 1669$ $(\mathrm{C}=\mathrm{O}), 1641$ $(\mathrm{C}=\mathrm{C}), 1569$

$(\mathrm{C}=\mathrm{N}), 1339,1130$ $\left(\mathrm{SO}_{2}\right)$

$3335(\mathrm{NH}), 1671$

$(\mathrm{C}=\mathrm{O}), 1645$

$(\mathrm{C}=\mathrm{C}), 1565$

$(\mathrm{C}=\mathrm{N}), 1333,1128$ $\left(\mathrm{SO}_{2}\right)$

$3328(\mathrm{NH}), 1682$ $(\mathrm{C}=\mathrm{O}), 1656$

$(\mathrm{C}=\mathrm{C}), 1571$

$(\mathrm{C}=\mathrm{N}), 1327,1138$ $\left(\mathrm{SO}_{2}\right)$

$3339(\mathrm{NH}), 1678$ $(\mathrm{C}=\mathrm{O}), 1651$

$9 b$

$(\mathrm{C}=\mathrm{C}), 1579$

$(\mathrm{C}=\mathrm{N}), 1321,1135$ $\left(\mathrm{SO}_{2}\right)$

$3332(\mathrm{NH}), 1684$

$(\mathrm{C}=\mathrm{O}), 1648$

$(\mathrm{C}=\mathrm{C}), 1583$

$(\mathrm{C}=\mathrm{N}), 1339,1145$

$\left(\mathrm{SO}_{2}\right)$ $5.22\left(\mathrm{~d}, 1 \mathrm{H}, \mathrm{C}_{4},-\mathrm{H}, J=6.4 \mathrm{~Hz}\right), 5.66\left(\mathrm{~d}, 1 \mathrm{H}, \mathrm{C}_{5},-\mathrm{H}, J=\right.$ $6.4 \mathrm{~Hz}), 6.93\left(\mathrm{~s}, 1 \mathrm{H}, \mathrm{C}_{2}-\mathrm{H}\right), 7.19-7.71(\mathrm{~m}, 15 \mathrm{H}, \mathrm{Ar} \&$ $\left.\mathrm{Ar}^{\prime}-\mathrm{H}\right), 8.01\left(\mathrm{~s}, 1 \mathrm{H}, \mathrm{C}_{5}-\mathrm{H}\right), 10.61$ (bs, $\left.1 \mathrm{H}, \mathrm{NH}\right)$

2.23 (s, 3H, Ar- $\left.\mathrm{CH}_{3}\right), 3.69$ (s, 3H, $\left.\mathrm{OCH}_{3}\right), 5.24(\mathrm{~d}, 1 \mathrm{H}$, $\left.\mathrm{C}_{4},-\mathrm{H}, J=6.5 \mathrm{~Hz}\right), 5.71\left(\mathrm{~d}, 1 \mathrm{H}, \mathrm{C}_{5},-\mathrm{H}, J=6.5 \mathrm{~Hz}\right)$, $6.98\left(\mathrm{~s}, 1 \mathrm{H}, \mathrm{C}_{2}-\mathrm{H}\right), 7.21-7.68$ (m, 13H, Ar \& Ar'-H), $7.99\left(\mathrm{~s}, 1 \mathrm{H}, \mathrm{C}_{5}-\mathrm{H}\right), 10.52(\mathrm{bs}, 1 \mathrm{H}, \mathrm{NH})$

$5.27\left(\mathrm{~d}, 1 \mathrm{H}, \mathrm{C}_{4},-\mathrm{H}, J=6.4 \mathrm{~Hz}\right), 5.76\left(\mathrm{~d}, 1 \mathrm{H}, \mathrm{C}_{5},-\mathrm{H}, J=\right.$ $6.4 \mathrm{~Hz}), 7.02\left(\mathrm{~s}, 1 \mathrm{H}, \mathrm{C}_{2}-\mathrm{H}\right), 7.25-7.78(\mathrm{~m}, 13 \mathrm{H}, \mathrm{Ar} \&$ Ar'-H), 8.06 (s, 1H, C $-\mathrm{H}$ ), 10.47 (bs, 1H, NH)

$6.38(\mathrm{bs}, 1 \mathrm{H}, \mathrm{NH}), 6.98\left(\mathrm{~s}, 1 \mathrm{H}, \mathrm{C}_{2}-\mathrm{H}\right), 7.26-7.62(\mathrm{~m}$, $\left.11 \mathrm{H}, \mathrm{C}_{5}{ }^{\prime}-\mathrm{H} \& \mathrm{Ar}-\mathrm{H}\right), 7.96\left(\mathrm{~s}, 1 \mathrm{H}, \mathrm{C}_{5}-\mathrm{H}\right), 8.84(\mathrm{bs}, 1 \mathrm{H}$, $\mathrm{NH})$

$2.31\left(\mathrm{~s}, 3 \mathrm{H}, \mathrm{Ar}_{-} \mathrm{CH}_{3}\right), 6.44$ (bs, $\left.1 \mathrm{H}, \mathrm{NH}\right), 7.01(\mathrm{~s}, 1 \mathrm{H}$, $\left.\mathrm{C}_{2}-\mathrm{H}\right), 7.28$ - $7.71\left(\mathrm{~m}, 10 \mathrm{H}, \mathrm{C}_{5}^{\prime}-\mathrm{H} \& \mathrm{Ar}-\mathrm{H}\right), 7.99(\mathrm{~s}$, $\left.1 \mathrm{H}, \mathrm{C}_{5}-\mathrm{H}\right), 8.72(\mathrm{bs}, 1 \mathrm{H}, \mathrm{NH})$

6.39 (bs, 1H, NH), 6.97 (s, 1H, $\left.\mathrm{C}_{2}-\mathrm{H}\right), 7.21$ - 7.78 (m, $\left.10 \mathrm{H}, \mathrm{C}_{5}-\mathrm{H} \& \mathrm{Ar}-\mathrm{H}\right), 8.03\left(\mathrm{~s}, 1 \mathrm{H}, \mathrm{C}_{5}-\mathrm{H}\right), 8.79(\mathrm{bs}, 1 \mathrm{H}$ $\mathrm{NH})$

7.04 (s, 1H, C2-H), 7.19 - 7.65 (m, 20H, Ar \& Ar'-H), $7.97\left(\mathrm{~s}, 1 \mathrm{H}, \mathrm{C}_{5}-\mathrm{H}\right), 10.46(\mathrm{bs}, 1 \mathrm{H}, \mathrm{NH})$

$2.29\left(\mathrm{~s}, 3 \mathrm{H}, \mathrm{Ar}-\mathrm{CH}_{3}\right), 3.71\left(\mathrm{~s}, 3 \mathrm{H}, \mathrm{OCH}_{3}\right), 6.98(\mathrm{~s}, 1 \mathrm{H}$ $\left.\mathrm{C}_{2}-\mathrm{H}\right), 7.22-7.71(\mathrm{~m}, 18 \mathrm{H}, \mathrm{Ar} \& \mathrm{Ar}-\mathrm{H}), 7.99(\mathrm{~s}, 1 \mathrm{H}$, $\left.\mathrm{C}_{5}-\mathrm{H}\right), 10.38$ (bs, $\left.1 \mathrm{H}, \mathrm{NH}\right)$

7.01 (s, 1H, C $2-\mathrm{H}), 7.27$ - 7.83 (m, 18H, Ar \& Ar'-H), $8.01\left(\mathrm{~s}, 1 \mathrm{H}, \mathrm{C}_{5}-\mathrm{H}\right), 10.42$ (bs, $\left.1 \mathrm{H}, \mathrm{NH}\right)$

$6.96\left(\mathrm{~s}, 1 \mathrm{H}, \mathrm{C}_{2}-\mathrm{H}\right), 7.09-7.68$ (m, 15H, Ar \& Ar'-H), $8.01\left(\mathrm{~s}, 1 \mathrm{H}, \mathrm{C}_{5}-\mathrm{H}\right), 10.48(\mathrm{bs}, 1 \mathrm{H}, \mathrm{NH})$

$2.26\left(\mathrm{~s}, 3 \mathrm{H}, \mathrm{Ar}-\mathrm{CH}_{3}\right), 3.67\left(\mathrm{~s}, 3 \mathrm{H},-\mathrm{OCH}_{3}\right), 6.92(\mathrm{~s}$, $\left.1 \mathrm{H}, \mathrm{C}_{2}-\mathrm{H}\right), 7.14$ - $7.76(\mathrm{~m}, 13 \mathrm{H}, \mathrm{Ar} \& \mathrm{Ar}-\mathrm{H}), 8.03$ (s, $\left.1 \mathrm{H}, \mathrm{C}_{5}-\mathrm{H}\right), 10.31(\mathrm{bs}, 1 \mathrm{H}, \mathrm{NH})$

7.03 (s, 1H, C $2-\mathrm{H}), 7.21$ - 7.82 (m, 13H, Ar \& Ar'-H), $7.99\left(\mathrm{~s}, 1 \mathrm{H}, \mathrm{C}_{5}-\mathrm{H}\right), 10.43(\mathrm{bs}, 1 \mathrm{H}, \mathrm{NH})$
62.4 (C-4'), 83.6 (C-5'), 108.7 (C-3), 116.9 (C-4), 122.5 (C-2), 136.8 (C-5), 155.0 (C-3'), $189.5(\mathrm{C}=\mathrm{O})$,

128.3, 129.1, 129.9, 130.2, 130.6, 131.2, 132.7, $133.4,134.6,136.3$ (aromatic carbons)

$21.7\left(\mathrm{Ar}^{-} \mathrm{CH}_{3}\right), 56.1\left(-\mathrm{OCH}_{3}\right), 63.1\left(\mathrm{C}-4{ }^{\prime}\right), 84.6$ (C-5'), 108.3 (C-3), 117.6 (C-4), 122.1 (C-2), 135.3 (C-5), $155.6(\mathrm{C}-3$ ') $188.3(\mathrm{C}=\mathrm{O}), 127.2,128.3$, $129.6,130.9,131.4,133.4,133.9,134.0,134.5$ (aromatic carbons)

62.7 (C-4'), 84.0 (C-5'), 108.9 (C-3), 116.5 (C-4), 122.9 (C-2), 134.9 (C-5), $154.2(\mathrm{C}-3$ '), $189.1(\mathrm{C}=\mathrm{O})$, $128.7,129.2,130.9,131.4,132.7,133.2,134.8$, 135.3, 137.2 (aromatic carbons)

110.1 (C-3), 115.9 (C-4), 122.1 (C-2), 135.2 (C-5), 137.3 (C-5'), 139.8 (C-4'), 153.4 (C-3'), 188.3 $(\mathrm{C}=\mathrm{O}), 128.1,129.7,130.7,131.1,132.4,133.9$, 134.2, 135.2 (aromatic carbons)

$22.7\left(\mathrm{Ar}^{-\mathrm{CH}_{3}}\right), 109.5$ (C-3), 115.1 (C-4), 122.9 (C-2), 134.8 (C-5), 136.6 (C-5'), 138.3 (C-4'), 154.7 (C-3'), $189.4(\mathrm{C}=\mathrm{O}), 128.6,129.3,130.1,131.8,132.7$, 133.2, 134.0, 134.9 (aromatic carbons)

110.4 (C-3), 114.7 (C-4), 122.2 (C-2), 135.0 (C-5), 135.1 (C-5'), 138.2 (C-4'), 155.2 (C-3'), 188.9 $(\mathrm{C}=\mathrm{O}), 127.4,128.7,130.6,131.2,132.3,133.5$, 134.6, 135.0, 135.6 (aromatic carbons)

109.9 (C-3), 116.4 (C-4), 121.9 (C-2), 136.9 (C-5), 146.5 (C-3'), 147.8 (C-4'), 153.2 (C-5'), 187.8 $(\mathrm{C}=\mathrm{O}), 127.0,127.9,128.7,129.2,129.9,130.9$,

131.8, 132.4, 133.9, 134.2, 135.3 (aromatic carbons)

$22.4\left(\mathrm{Ar}^{\left.-\mathrm{CH}_{3}\right)}, 56.6\left(-\mathrm{OCH}_{3}\right), 109.2(\mathrm{C}-3), 116.9\right.$ (C-4), 122.6 (C-2), 136.2 (C-5), 146.9 (C-3'), 148.2 (C-4'), 152.9 (C-5'), 188.5 (C=O), 128.2, 129.1, $129.7,130.2,131.5,132.9,133.5,134.0,134.7$ (aromatic carbons)

109.5 (C-3), 116.1 (C-4), 121.8 (C-2), 136.4 (C-5), 146.1 (C-3'), 148.9 (C-4'), 152.2 (C-5'), 189.4 $(\mathrm{C}=\mathrm{O}), 128.7,129.4,129.9,130.5,131.9,132.2$, 133.3, 134.6, 135.9 (aromatic carbons)

108.4 (C-3), 116.1 (C-4), 119.3 (C-2), 138.4 (C-5), 147.1 (C-4'), 148.3 (C-3'), 151.8 (C-5'), 187.7 $(\mathrm{C}=\mathrm{O}), 130.0,130.3,130.4,130.6,130.8,131.0$, 132.7, 134.1 (aromatic carbons)

$22.7\left(\mathrm{Ar}-\mathrm{CH}_{3}\right), 57.8\left(-\mathrm{OCH}_{3}\right), 109.1(\mathrm{C}-3), 117.2$ (C-4), 122.9 (C-2), 135.7 (C-5), 146.9 (C-4'), 148.8 (C-3'), 152.9 (C-5'), 189.9 (C=O), 127.6, 128.2, $128.7,130.7,131.3,131.9,132.8,133.1,134.8$ (aromatic carbons)

109.6 (C-3), 117.9 (C-4), 122.4 (C-2), 135.9 (C-5), 146.2 (C-4'), 148.3 (C-3'), 153.4 (C-5'), 189.7 $(\mathrm{C}=\mathrm{O}), 128.6,129.5,130.4,131.2,132.6,133.1$, $133.9,134.7,135.9$ (aromatic carbons) 
and $J_{\mathrm{MX}}=10.3 \mathrm{~Hz}$ indicates that $\mathrm{H}_{\mathrm{A}}$ and $\mathrm{H}_{\mathrm{M}}$ are cis, $\mathrm{H}_{\mathrm{A}}$ and $\mathrm{H}_{\mathrm{X}}$ are trans and $\mathrm{H}_{\mathrm{M}}$ and $\mathrm{H}_{\mathrm{X}}$ are geminal (Table 2). Similarly, 1,3-dipolar cycloaddition reaction of nitrile imines and nitrile oxides generated from araldehyde phenylhydrazones and araldoximes to 2 resulted in 4aroyl-3-(1',5'-diphenyl-3'-aryl-4',5'-dihydro-1' $H$-pyrazo 1-4'-ylsulfonyl)-1H-pyrrole (5) and 4-aroyl-3-(3'-aryl5'-phenyl-4',5'-dihydroisoxazol-4'-ylsulfonyl)- $1 H$-pyrro le (6), respectively (Scheme 1, Table 1). The ${ }^{1} \mathrm{H}-\mathrm{NMR}$ spectra of 5a and 6a displayed two doublets at $\delta 5.19$, 5.22 and 5.58, $5.66 \mathrm{ppm}$, which are assigned to $\mathrm{C}_{4},-\mathrm{H}$ and $\mathrm{C}_{5},-\mathrm{H}$, the two methine protons of the pyrazoline and isoxazoline rings. The $J$ values $(J=6.3 \& 6.4 \mathrm{~Hz})$ shows that they are in trans geometry.

The compounds 4, 5 and 6 upon oxidation with chloranil in xylene gave the corresponding pyrazoles and isoxazoles, 4-aroyl-3-(4'-phenyl-1' $H$-pyrazol-3'-ylsulfonyl)-1H-pyrrole (7), 4-aroyl-3-(1',5'-diphenyl-3'-aryl1 ' $H$-pyrazol-4'-ylsulfonyl)-1 $H$-pyrrole (8) and 4-aroyl3-(3'-aryl-5' -phenylisoxazol-4' -ylsulfonyl)-1 $H$-pyrrole (9) (Scheme 1, Table 1). The disappearance of signals due to pyrazoline/isoxazoline ring protons in the ${ }^{1} \mathrm{H}$ NMR spectra of 7-9 confirms their formation. The structures of 2-9 are further established by elemental analyses, IR and ${ }^{13} \mathrm{C}$ NMR spectroscopy (Tables 1 and 2).

\section{Antimicrobial Testing}

The compounds 2, 3, 7-9 were tested for antimicrobial activity at two different concentrations 100 and 200 $\mu \mathrm{g} / \mathrm{mL}$. The antibacterial activity was screened against Staphylococcus aureus, Bacillus subtilis (Gram-positive bacteria) and Escherichia coli, Klebsiella pneumoniae (Gram-negative bacteria) on nutrient agar plates at $37^{\circ} \mathrm{C}$ for $24 \mathrm{hr}$ using chloramphenicol as reference drug. The compounds were also evaluated for their antifungal activity against Fusarium solani, Curvularia lunata and Aspergillus niger using ketoconazole as standard drug. Fungi cultures were grown on potato dextrose agar medium (PDA) at $25^{\circ} \mathrm{C}$ for 3 days. The spore suspension was adjusted to 106 pores $/ \mathrm{mL}$ at an $\mathrm{mg} / \mathrm{mL}$ concentration by the Vincent and Vincent method [23].

The results of the compounds of preliminary antibacterial testing are shown in Table 3 . The results revealed that the compounds $\mathbf{2}$ and $\mathbf{3}$ exhibited least activity against Gram-positive bacteria and almost no activity against Gram-negative bacteria. However, the other compounds showed higher inhibitory activity against Grampositive bacteria than that of Gram-negative bacteria. It was reported that good DNA binding properties are a prerequisite for antibacterial activity [8]. This was evidenced by the fact that the compounds 7 showed good activity when compared with compounds $\mathbf{8}$. This may be due to the bulkier tetrasubstituted pyrazole destroys DNA binding and activity. In fact, the compounds having trisubstituted pyrazole (7) and disubstituted isoxazole (9) units exhibited good activity when compared with the compounds having terasubstituted pyrazole (8) unit. It was observed that the presence of chloro substituent enhances the activity. The compounds $7 \mathbf{c}$ and 9c displayed excellent activity against Gram-positive bacteria (inhibitory zone $>28 \mathrm{~mm}$ ) and good activity against Gramnegative bacteria (inhibitory zone $>22 \mathrm{~mm}$ ). All the test compounds showed moderate to high inhibitory effect towards tested fungi (Table 4).

The MIC values were determined as the lowest concentration that completely inhibited visible growth of the microorganisms (Table 5). The structure-antimicrobial activity relationship of the tested compounds revealed that disubstituted pyrazole and trisubstituted isoxazole in

Table 3. The in vitro antibacterial activity of compounds 2, 3, 7-9.

\begin{tabular}{|c|c|c|c|c|c|}
\hline \multirow{3}{*}{ Compd } & \multirow{3}{*}{$\begin{array}{l}\text { Concentration } \\
\qquad(\mu \mathrm{g} / \mathrm{ml})\end{array}$} & \multicolumn{4}{|c|}{ Zone of inhibition (mm) } \\
\hline & & \multicolumn{2}{|c|}{ Gram (+)ve } & \multicolumn{2}{|c|}{ Gram (-)ve } \\
\hline & & $\begin{array}{c}\text { S. } \\
\text { aureus }\end{array}$ & $\begin{array}{l}\text { B. } \\
\text { ubtilis }\end{array}$ & $\begin{array}{c}E . \\
\text { coli }\end{array}$ & $\begin{array}{c}\text { K. } \\
\text { pneumoniae }\end{array}$ \\
\hline \multirow[t]{2}{*}{$2 \mathbf{a}$} & 100 & 10 & 9 & - & - \\
\hline & 200 & 12 & 11 & - & - \\
\hline \multirow[t]{2}{*}{$2 b$} & 100 & 8 & 9 & - & - \\
\hline & 200 & 10 & 11 & - & - \\
\hline \multirow[t]{2}{*}{ 2c } & 100 & 13 & 11 & 18 & 9 \\
\hline & 200 & 15 & 14 & 19 & 11 \\
\hline \multirow[t]{2}{*}{$3 \mathbf{a}$} & 100 & 12 & 13 & - & - \\
\hline & 200 & 15 & 15 & 10 & - \\
\hline \multirow[t]{2}{*}{$\mathbf{3 b}$} & 100 & 10 & 09 & - & - \\
\hline & 200 & 12 & 12 & - & - \\
\hline \multirow[t]{2}{*}{$3 c$} & 100 & 15 & 14 & 11 & 12 \\
\hline & 200 & 18 & 17 & 13 & 14 \\
\hline \multirow[t]{2}{*}{$7 \mathbf{a}$} & 100 & 25 & 22 & 19 & 18 \\
\hline & 200 & 28 & 24 & 22 & 20 \\
\hline \multirow[t]{2}{*}{$7 \mathbf{b}$} & 100 & 19 & 20 & 18 & 16 \\
\hline & 200 & 21 & 23 & 20 & 19 \\
\hline \multirow[t]{2}{*}{ 7c } & 100 & 30 & 28 & 22 & 20 \\
\hline & 200 & 32 & 31 & 25 & 23 \\
\hline \multirow[t]{2}{*}{ 8a } & 100 & 16 & 15 & 15 & 14 \\
\hline & 200 & 18 & 17 & 17 & 17 \\
\hline \multirow[t]{2}{*}{$\mathbf{8 b}$} & 100 & 15 & 16 & 10 & 12 \\
\hline & 200 & 17 & 18 & 13 & 14 \\
\hline \multirow[t]{2}{*}{$8 c$} & 100 & 19 & 19 & 15 & 14 \\
\hline & 200 & 21 & 21 & 17 & 17 \\
\hline \multirow[t]{2}{*}{ 9a } & 100 & 23 & 21 & 18 & 18 \\
\hline & 200 & 25 & 24 & 20 & 19 \\
\hline \multirow[t]{2}{*}{$9 b$} & 100 & 22 & 20 & 17 & 15 \\
\hline & 200 & 24 & 23 & 19 & 17 \\
\hline \multirow[t]{2}{*}{$9 c$} & 100 & 27 & 25 & 21 & 20 \\
\hline & 200 & 29 & 28 & 24 & 23 \\
\hline \multirow[t]{2}{*}{ Chloraphenicol } & 100 & 35 & 38 & 37 & 42 \\
\hline & 200 & 41 & 44 & 42 & 45 \\
\hline
\end{tabular}


Table 4. The in vitro antifungal activity of compounds 2, 3, 7-9.

\begin{tabular}{|c|c|c|c|c|}
\hline \multirow{2}{*}{ Compd } & \multirow{2}{*}{$\begin{array}{l}\text { Concentration } \\
(\mu \mathrm{g} / \mathrm{ml})\end{array}$} & \multicolumn{3}{|c|}{ Zone of inhibition (mm) } \\
\hline & & F. solani & C. lunata & A. niger \\
\hline \multirow[t]{2}{*}{$2 a$} & 100 & 17 & 13 & 12 \\
\hline & 200 & 21 & 16 & 14 \\
\hline \multirow[t]{2}{*}{$2 \mathbf{b}$} & 100 & 15 & 12 & 10 \\
\hline & 200 & 18 & 14 & 13 \\
\hline \multirow[t]{2}{*}{ 2c } & 100 & 17 & 17 & 15 \\
\hline & 200 & 20 & 21 & 19 \\
\hline \multirow[t]{2}{*}{ 3a } & 100 & 16 & 13 & 10 \\
\hline & 200 & 18 & 14 & 13 \\
\hline \multirow[t]{2}{*}{$3 \mathbf{b}$} & 100 & 14 & 10 & 9 \\
\hline & 200 & 15 & 12 & 12 \\
\hline \multirow[t]{2}{*}{$3 c$} & 100 & 18 & 16 & 14 \\
\hline & 200 & 20 & 19 & 17 \\
\hline \multirow[t]{2}{*}{$7 \mathbf{a}$} & 100 & 29 & 26 & 22 \\
\hline & 200 & 32 & 32 & 24 \\
\hline \multirow[t]{2}{*}{$7 \mathbf{b}$} & 100 & 26 & 26 & 23 \\
\hline & 200 & 30 & 31 & 26 \\
\hline \multirow[t]{2}{*}{ 7c } & 100 & 33 & 32 & 27 \\
\hline & 200 & 35 & 36 & 29 \\
\hline \multirow[t]{2}{*}{$\mathbf{8 a}$} & 100 & 17 & 17 & 14 \\
\hline & 200 & 20 & 21 & 17 \\
\hline \multirow[t]{2}{*}{$8 \mathbf{b}$} & 100 & 16 & 16 & 15 \\
\hline & 200 & 19 & 20 & 18 \\
\hline \multirow[t]{2}{*}{$8 \mathrm{c}$} & 100 & 17 & 18 & 17 \\
\hline & 200 & 21 & 21 & 20 \\
\hline \multirow[t]{2}{*}{ 9a } & 100 & 25 & 26 & 23 \\
\hline & 200 & 27 & 29 & 26 \\
\hline \multirow[t]{2}{*}{$9 b$} & 100 & 22 & 23 & 19 \\
\hline & 200 & 26 & 25 & 23 \\
\hline \multirow[t]{2}{*}{ 9c } & 100 & 28 & 28 & 26 \\
\hline & 200 & 32 & 30 & 29 \\
\hline \multirow[t]{2}{*}{ Ketoconazole } & 100 & 38 & 41 & 36 \\
\hline & 200 & 42 & 44 & 39 \\
\hline
\end{tabular}

combination with pyrrole displayed greater activity. The compounds having tetrasubstituted pyrazole with pyrrole exhibited least activity. The maximum activity wasobserved with the compounds $\mathbf{7 c}$ and $\mathbf{9 c}$.

\section{Experimental Section}

Melting points were determined in open glass capillaries on a Mel-Temp apparatus and are uncorrected. The purity of the compounds was checked by TLC (silica gel H, BDH, ethyl acetate/hexane, 1:3). The IR spectra were recorded on a Thermo Nicolet IR 200 FT-IR spectrometer as $\mathrm{KBr}$ pellets and the wave numbers were given in $\mathrm{cm}^{-1}$. The ${ }^{1} \mathrm{H}$ and ${ }^{13} \mathrm{C}$ NMR spectra were run in $\mathrm{CDCl}_{3} /$ DMSO- $d_{6}$ on a Jeol JNM spectrometer operating at 400 and $100 \mathrm{MHz}$. All chemical shifts were reported in $\delta$ ppm using TMS as an internal standard. The elemental analyses were determined on a Perkin-Elmer $24^{\circ} \mathrm{C}$ elemental analyzer. The starting material 1-aroyl-2-styrylsulfonylethene (1) was prepared by the literature procedure [24].

Method 1:

General procedure for the synthesis of 4-aroyl-3-(phenylethenesulfonyl)- $1 H$-pyrrole (2)/4-aroyl-3-(4'-phenyl1' $H$-pyrrol-3'-ylsulfonyl)- $1 H$-pyrrole (3)

A mixture of $1(0.5 \mathrm{mmol})$ and TosMIC $(1 \mathrm{mmol})$ in $\mathrm{Et}_{2} \mathrm{O}$-DMSO (2:1) was added dropwise under stirring to a suspension of $\mathrm{NaH}(2 \mathrm{mmol})$ in $\mathrm{Et}_{2} \mathrm{O}(10 \mathrm{~mL})$ at room temperature and stirring was continued for $5-6 \mathrm{hr}$. Then, water was added and the reaction mass was extracted with $\mathrm{Et}_{2} \mathrm{O}$. The ethereal fraction was dried over anhydrous $\mathrm{Na}_{2} \mathrm{SO}_{4}$. The solvent was removed in vacuo. The resulting mixture was separated by column chromatography (hexane-ethyl acetate; 4:1) and identified as 4-aroyl-3-(phenylethenesulfonyl)-1 $H$-pyrrole (2) (major) and 4-aroyl-3-(4'-phenyl-1' $H$-pyrrole-3'-ylsulfonyl)- $1 H$ pyrrole (3) (minor).

Method 2:

General procedure for the synthesis of 4-aroyl-3-(4'phenyl-1' $H$-pyrrol-3'-ylsulfonyl)-1 $H$-pyrrole (3)

A solution of $1(1 \mathrm{mmol})$ and TosMIC $(4 \mathrm{mmol})$ in $\mathrm{Et}_{2} \mathrm{O}$-DMSO (2:1) was added dropwise under stirring to a suspension of $\mathrm{NaH}(4 \mathrm{mmol})$ in $\mathrm{Et}_{2} \mathrm{O}(20 \mathrm{~mL})$ at $\mathrm{RT}$ and stirring was continued for about 3 - $4 \mathrm{hr}$. Then, water was added and the reaction mass extracted with $\mathrm{Et}_{2} \mathrm{O}$. The ethereal layer was dried (an. $\mathrm{Na}_{2} \mathrm{SO}_{4}$ ) and the solvent was removed in vacuo. The solid obtained was purified by column chromatography (ethyl acetate/hexane, 1:4).

\section{Method 3:}

The compound 3 was also obtained by adding an equimolar $(5 \mathrm{mmol})$ mixture of 2 and TosMIC in $\mathrm{Et}_{2} \mathrm{O}$-DMSO (2:1) dropwise under stirring to a suspension of $\mathrm{NaH}(1 \mathrm{mmol})$ in $\mathrm{Et}_{2} \mathrm{O}(6 \mathrm{~mL})$ at RT. Stirring was continued for $4-5 \mathrm{hr}$. Then, the contents were diluted with water and extracted with $\mathrm{Et}_{2} \mathrm{O}$. The ethereal layer was dried over anhydrous $\mathrm{Na}_{2} \mathrm{SO}_{4}$. Evaporation of the solvent

Table 5. Minimum inhibitory concentration of compounds 7c and 9c.

\begin{tabular}{|c|c|c|c|c|c|c|c|}
\hline \multirow{2}{*}{ Compd } & \multicolumn{7}{|c|}{ Minimum inhibitory concentration (MIC), $\mu \mathrm{g} / \mathrm{ml}$} \\
\hline & S. aureus & B. subtilis & E. Coli & K. pneumoniae & F. solani & C. lunata & A. niger \\
\hline 7c & 50 & 50 & 100 & 50 & 25 & 12.5 & 50 \\
\hline Chloramphenicol & 6.25 & 6.25 & 6.25 & 12.5 & - & - & - \\
\hline Ketoconazole & - & - & - & - & 12.5 & 6.25 & 6.25 \\
\hline
\end{tabular}


under vacuum resulted in a solid which was purified by column chromatography (ethyl acetate/hexane, 1:4).

General procedure for the synthesis of 4-aroyl-3-(4'phenyl-4',5'-dihydro-1' $H$-pyrazol-3'-ylsulfonyl)-1 $H$-pyr role (4)

To a cooled solution of $2(5 \mathrm{mmol})$ in dichloromethane $(20 \mathrm{~mL})$, an ethereal solution of diazomethane $(40 \mathrm{ml}$, $0.4 \mathrm{M})$ and triethylamine $(0.12 \mathrm{~g})$ were added. The reaction mixture was kept at -20 to $-15{ }^{\circ} \mathrm{C}$ for $40-48 \mathrm{hr}$. The solvent was removed under reduced pressure and the resultant solid was recrystallized from 2-pro- panol.

General procedure for the synthesis of 4-aroyl-3(1',5'-diphenyl-3' -aryl-4',5' -dihydro-1' H-pyrazol-4-ylsulfonyl)- $1 H$-pyrrole (5)

A mixture of 2 (1 mmol), araldehyde phenylhydrazone (2 mmol) and chloramine-T (2 mmol) in methanol (15 $\mathrm{mL}$ ) was refluxed for $16-18 \mathrm{hr}$ on a water bath. The precipitated inorganic salts were filtered off. The filtrate was concentrated and the residue was extracted with dichloromethane. The organic layer was washed with water, saturated brine and dried over anhydrous $\mathrm{Na}_{2} \mathrm{SO}_{4}$. Evaporation of the solvent under reduced pressure afforded a crude product which was recrystallized from ethanol.

General procedure for the synthesis of 4-aroyl-3(3'-aryl-5' -phenyl-4',5'-dihydroisoxazol-4'-ylsulfonyl)$1 H$-pyrrole (6)

The compound $2(1 \mathrm{mmol})$, araldoxime $(2 \mathrm{mmol})$ and chloramine-T $(2 \mathrm{mmol})$ in methanol $(20 \mathrm{~mL})$ was refluxed for 14-16 $\mathrm{hr}$ on a water bath. The precipitated inorganic salts were filtered off. The filtrate was concentrated and the residue was extracted with dichloromethane. The organic layer was washed with water, saturated brine and dried over anhydrous $\mathrm{Na}_{2} \mathrm{SO}_{4}$. The solvent was removed in vacuo. The solid obtained was purified by recrystallization from ethanol.

General procedure for the synthesis of 4-aroyl-3(4'-phenyl-1' $H$-pyrazol-3'-ylsulfonyl)-1 $H$-pyrrole (7)/4aroyl-3-(1',5'-diphenyl-3'-aryl-1' $H$-pyrazol-4'-yl-sulfon yl)-1H-pyrrole (8)/4-aroyl-3-(3'-aryl-5' -phenylisoxazol4'-ylsulfonyl)-1 $H$-pyrrole (9)

A solution of 4-6 (1 mmol) and chloranil $(1.4 \mathrm{mmol})$ in xylene $(10 \mathrm{~mL})$ was refluxed for $25-30 \mathrm{hr}$. Then, the reaction mixture was treated with a $5 \% \mathrm{NaOH}$ solution. The organic layer was separated and repeatedly washed with water. It was then dried over anhydrous $\mathrm{Na}_{2} \mathrm{SO}_{4}$ and the solvent was removed on a rotary evaporator. The resultant solid was purified by recrystallization from methanol.

\section{Conclusions}

A new class of bis heterocycles 4-aroyl-3-(4'-phenyl-
1'H-pyrazol-3'-ylsulfonyl)-1H-pyrrole (7), 4-aro-yl-3(1',5'-diphenyl-3' -aryl-1' $H$-pyrazol-4'-ylsulfonyl)- $1 H$ pyrrole (8) and 4-aroyl-3-(3'-aryl-5'-phenylisoxazol4'-ylsulfonyl)-1H-pyrrole (9) were prepared by the regioselective reaction of tosylmethyl isocyanide and 1,3dipolar cycloaddtion reaction of diazomethane, nitrile imines and nitrile oxides with 1-aroyl-2-styrylsulfonylethene (1). The antimicrobial testing showed that the compounds 7c and 9c exhibited greater antimicrobial activity.

\section{Acknowledgements}

The authors are thankful to UGC, New Delhi, India for financial assistance under major research project.

\section{References}

[1] S. S. Parmar, B. R. Pandey, C. Dwivedi and R. D. Harbison, "Anticonvulsant Activity and Monoamine Oxidase Inhibitory Properties of 1,3,5-Trisubstituted Pyrazolines," Journal of Pharmaceutical Sciences, Vol. 63, No. 7, 1974, pp. 1152-1155.doi:10.1002/jps. 2600630730

[2] N. Soni, K. Pande, R. Kalsi, T. K. Gupta, S. S. Parmar and J. P. Barthwal, "Evaluation of Anti-Depressant-Like Effect of 2-Pyrazoline," Research Communications in Chemical Pathology and Pharmacology, Vol. 56, 1987, pp. 129-132.

[3] G. Dannahardt, W. Kiefer, G. Kramer, S. Maehrlein, U. Nowe and B. Fiebich, "The Pyrrole Moiety as a Template for COX-1/COX-2 Inhibitors," European Journal of Medicinal Chemistry, Vol. 35, No. 5, 2000, pp. 499-510. doi:10.1016/S0223-5234(00)00150-1

[4] C. C. C. Wang and P. B. Derven, "Sequence-Specific Trapping of Topoisomerase I by DNA Binding Polyamide-Camptothecin Conjugates," Journal of the American Chemical Society, Vol. 123, No. 36, 2001, pp. 8657-8661. doi:10.1021/ja010392p

[5] B. Wellenzohn, W. Flader, R. H. Winger, A. Hallabruker, E. Mayer and K. R. Liedl, "Complex of B-DNA with Polyamides Freezes DNA Backbone Flexibility," Journal of the American Chemical Society, Vol. 123, 2001, pp. 5044-5049. doi:10.1021/ja003639b

[6] S. K. Sharma, M. Tandon and J. W. Lown, "A General Solution and Solid-Phase Synthetic Procedure for Incorporating Three Contiguous Imidazole Moieties into DNA Sequence Reading Polyamides," Journal of Organic Chemistry, Vol. 66, No. 3, 2001, pp. 1030-1034. doi:10.1021/jo001034s

[7] N. R. Wurtz, J. M. Turner, E. E. Baird and P. B. Dervan, "Solid phase synthesis of polyamides containing pyrrole and imidazole amino acids," Organic Letters, Vol. 3, No. 8, 2001, pp. 1201-1203. doi:10.1021/o10156796

[8] N. B. Dyatkina, C. D. Roberts, J. D. Keicher, Y. Dai, J. P. Nadherny, W. Zhang, U. Schmitz, A. Kongpachith, K. Fung, A. A. Nokikov, L. Lou, M. Velligan, A. A. Khor- 
lin and M. S. Chen, "Minor Groove DNA Binders as Antimicrobial Agents. 1. Pyrrole Tetraamides Are Potent Antibacterials against Vancomycin Resistant Enteroccoci and Methicillin Resistant," Journal of Medicinal Chemistry, Vol. 45, No. 4, 2002, pp. 805-817. doi:10.1021/jm010375a

[9] M. Curini, F. Montanari, O. Rosati, E. Lioy and R. Margarita, "Layered Zirconium Phosphate and Phosphonate as Heterogeneous Catalyst in the Preparation of Pyrroles," Tetrahedron Letters, Vol. 44, No. 20, 2003, pp. 3923-3925.doi:10.1016/S0040-4039(03)00810-4

[10] A. M. Van Leusen, H. Siderius, B. E. Hoogenboom and D. van Leusen, "A New and Simple Synthesis of the Pyrrole Ring System from Michael Acceptors and Tosylmethylisocyanides," Tetrahedron Letters, Vol. 13, No. 52, 1972, pp. 5337-5340. doi:10.1016/S0040-4039(01)85244-8

[11] N. P. Pavri and M. L. Trudell, "An Efficient Method for the Synthesis of 3-Arylpyrroles," Journal of Organic Chemistry, Vol. 62, No. 8, 1997, pp. 2649-2651. doi:10.1021/jo961981u

[12] T. N. Danks, "Microwave Assisted Synthesis of Pyrroles," Tetrahedron Letters, Vol. 40, No. 20, 1999, pp. 3957- 3960. doi:10.1016/S0040-4039(99)00620-6

[13] G. Minetto, L. F. Raveglia and M. Taddei, "Microwave-Assisted Paal-Knorr Reaction. A Rapid Approach to Substituted Pyrroles and Furans," Organic Letters, Vol. 6, No. 3, 2004, pp. 389-392. doi:10.1021/ol0362820

[14] A. G. Lee, "A Simplified Synthesis of Unsaturated Nitrogen-Heterocycles Using Nitrile Betaines," Synthesis, 1982, pp. 508-509. doi:10.1055/s-1982-29860

[15] Z. Bao-Xiang, Yu Yang and E. Shoji, "Synthesis of Stable $\Delta^{4}$-Isoxazolines by 1,3-Dipolar Cycloaddition of 3,4-Dihydroisoquinoline $N$-Oxides with Alkynes and Their Rearrangement to Isoquinoline-Fused Pyrroles," Tetrahedron, Vol. 52, No. 37, 1996, pp. 12049-12060. doi:10.1016/0040-4020(96)00698-9

[16] G. Just and K. Dhal, "Lead Tetraacetate Oxidation of
Aldoximes," Tetrahedron, Vol. 24, No. 15, 1968, pp. 5251-5269. doi:10.1016/S0040-4020(01)96322-7

[17] K. M. L. Rai, N. Linganna, A. Hassner and C. A. Murthy, "A Convenient Method for the Generation of Nitrile Oxide and Its Application to the Synthesis of 2-Isoxazolines," Organic Preparations and Procedures International, Vol. 24, 1992, pp. 91-94. doi:10.1080/00304949209356711

[18] J. N. Kim and E. K. Ryu, "A Convenient Synthesis of Nitrile Oxides from Aldoximes by 1-Chloroben Zotriazole," Synthetic Communications, Vol. 20, No. 9, 1990, pp. 1373-1377. doi:10.1080/00397919008052851

[19] K. M. L. Rai and A. Hassner, "Intramolecular 1,3-Diploar cycoaddition of Nitrile Oxides with Vinyl Acetate and Acrylonitrile," Indian Journal of Chemistry, Vol. 36B, 1997, pp. 242-245.

[20] K. M. L. Rai and A. Hassner, "Intermolecular 1,3-Dipolar cycloadditions of Preformed Nitrile Oxides with Phenyl Vinyl Sulphone," Synthetic Communications, Vol. 27, 1997, pp. 467-472. doi:10.1080/00397919708006048

[21] A. Hassner and K. M. Lokanath Rai, "A New Method for the Generation of Nitrile Oxides and Its Application to the Synthesis of 2-Isoxazolines," Synthesis, Vol. 1, 1989, pp. 57-59. doi:10.1055/s-1989-27152

[22] K. M. L. Rai and A. Hassner, "Chlorahine-T in Heterocyclic Synthesis; a Simple Procedure for the Genedation of Nitrilihines and Its Application to the Synthesis of Pyrazolines," Synthetic Communications, Vol. 19, No. 16, 1989, pp. 2799-2807. doi:10.1080/00397918908052667

[23] J. G. Vincent and H. W. Vincent, "Filter Paper Disc Modification of the Oxford Cup Penicillin Determination," Proceedings of the Society for Experimental Biology and Medicine, Vol. 55, No. 3, 1944, pp. 162-164.

[24] D. B. Reddy, N. C. Babu, K. V. Reddy and V. Padmavathi, "A New Route for the Synthesis of Bisunsaturated Oxosulfones and Bis Sulfones under FriedelCrafts Conditions," Indian Journal of Chemistry, Vol. 40B, 2001, pp. 416-418. 